\title{
Clinical characteristics and epilepsy outcomes following surgery caused by focal cortical dysplasia (type IIa) in 110 adult epileptic patients
}

\author{
YUQIANG SUN ${ }^{1,2 *}$, XIAOFENG WANG ${ }^{3 *}$, NINGWEI CHE ${ }^{1}$, HUAMIN QIN $^{4}$, SHUPING LIU $^{1}$, \\ XINLING WU ${ }^{5}$, MINGHAI WEI ${ }^{1}$, HUAKUN CHENG ${ }^{2}$ and JIAN YIN ${ }^{1}$
}

\author{
${ }^{1}$ Department of Neurosurgery, The Second Affiliated Hospital of Dalian Medical University, Dalian, Liaoning 116044; \\ ${ }^{2}$ Department of Neurosurgery, Heilongjiang Provincial Hospital, Harbin, Heilongjiang 150036; ${ }^{3}$ Department of Neurosurgery, \\ Weinan Central Hospital, Weinan, Shaanxi 714000; ${ }^{4}$ Department of Pathology, The Second Affiliated Hospital of \\ Dalian Medical University; ${ }^{5}$ Department of Psychology, Dalian Medical University, Dalian, Liaoning 116044, P.R. China
}

Received August 9, 2015; Accepted October 21, 2016

DOI: $10.3892 / \mathrm{etm} .2017 .4315$

\begin{abstract}
The aim of the present study was to investigate the effects of surgical intervention of focal cortical dysplasia (FCD) IIa on the outcome of epilepsy, and to evaluate the prognostic factors of seizure freedom. Patient data from epilepsy surgeries were retrospectively reviewed at the Second Affiliated Hospital of Dalian Medical University between 2007 and 2015. A total of 110 patients with a definite pathological diagnosis of FCD IIa were included. Moreover, the clinical characteristics, seizure outcome and quality of life in adults with FCD IIa were evaluated. The Engel seizure outcome achievements were class I in 72, class II in 20, class III in 11 and class IV in 7 patients. In addition, the Engel seizure outcome was relevant with the resection range of the lesions $(\mathrm{P}=0.028)$. The assessments of electrocorticography $(\mathrm{ECoG})$ patterns and magnetic resonance imaging (MRI) are relevant to determining the extent of the resection, which may influence the surgery outcome $(\mathrm{P}=0.001$ and $\mathrm{P}=0.023)$. Using multivariate regression analyses, the extent of resection, seizure frequency, preoperative ECoG and location of resection were the most important risk factors for seizure recurrence. The results of quality of life in epilepsy-10 scoring revealed that the quality
\end{abstract}

Correspondence to: Dr Jian Yin, Department of Neurosurgery, The Second Affiliated Hospital of Dalian Medical University, 467 Zhongshan Road, Dalian, Liaoning 116044, P.R. China

E-mail: dryin163@163.com

Dr Huakun Cheng, Department of Neurosurgery, Heilongjiang Provincial Hospital, 82 Zhongshan Road, Xiangfang, Harbin, Heilongjiang 150036, P.R. China

E-mail: weiminghai1765@163.com

\section{*Contributed equally}

Key words: focal cortical dysplasia, adult epilepsy, surgery outcome, clinical characteristics, quality of life of life improved significantly following surgery $(\mathrm{P}<0.01)$. Moreover, surgical intervention, EcoG, MRI positioning and complete resection helped to have improved seizure control, relief of anxiety and quality of life. All these observations strongly recommend an early consideration of epilepsy surgery in FCD IIa patients.

\section{Introduction}

Focal cortical dysplasia (FCD) was initially described by Taylor et al (1) and is one of the most common causes of refractory epilepsy (2). FCDs are localized regions of malformed cerebral cortex and are often associated with epilepsy in both adults and children (3). FCDs are recognized by their histological attributes, which include blurring of the cortical dyslamination, gray-white matter junction, and/or the presence of abnormal balloon cells in the cortical and subcortical regions (4). A broad spectrum of histopathology has been included in the diagnosis of FCD, and the classification method created by Blümcke et al (5) has been applied more widely. The classification system included aberrant radial or tangential lamination of the neocortex (FCD Type I), cytological abnormalities (FCD Type II) and cortical dyslamination with hippocampal sclerosis, epilepsy-associated tumours, vascular malformations and epileptogenic lesions acquired in early life (FCD, Type III). In recent years, the number of patients with FCD IIa in the Second Affiliated Hospital of Dalian Medical University (Dalian, China) has significantly increased. The characteristic of the FCD IIa is the presence of dysmorphic neurons, which have an enlarged cell body and nucleus, abnormally distributed intracellular Nissl substance and cytoplasmic accumulation of neurofilament proteins (4). However, no balloon cells were observed.

In the majority of patients with epilepsy, the disease became refractory to medical therapy (6). Therefore, surgical intervention is usually necessary. However, previous results have documented unsatisfactory epilepsy surgery outcomes in this group of patients (7). Due to the progress in neuroimaging, including magnetic resonance imaging (MRI) and 
electrocorticography (ECoG), the FCD becomes more visible to delineate in preoperative assessment, and therefore the use of surgery is once again increasing in recent years $(8,9)$. The outcome of surgery for epilepsy has not been studied well and there are few long-term data that are relevant to the seizure outcome. The identification of risk factors for seizure recurrence following epilepsy surgery for seizures is important for preoperative and postoperative counseling and evaluation (10). The challenge is to remove the whole lesion, since complete resection has proven to be an essential prognostic factor of seizure freedom $(11,12)$. Determination of prognostic factors for surgery for epilepsy is important when counselling patients (13). Moreover, identification of prognostic factors may improve the general understanding of the disease. Hence, the purpose of the present study is to evaluate prognostic factors that influence the epilepsy outcome by comparing the changes of clinical characteristics, electroencephalogram (EEG), MRI and the quality of life prior to and following the surgery.

Surgical resection has been an important alternative treatment for patients with intractable epilepsy. Preoperative and intraoperative ECoG provides a unique opportunity to assess the epileptogenicity of exposed cortical areas during surgery (14). Although the EEG is important in the diagnosis and prediction of the outcome of surgery for epilepsy, the surgical outcome in patients with MRI abnormalities is satisfactory compared to patients without an identifiable lesion on presurgical MRI. Localization using MRI and ECoG can carry an important prognostic value due to patients with identifiable, well-defined lesions being more likely to have satisfactory postoperative seizure-free outcomes $(15,16)$. Moreover, MRI results are abnormal in $\sim 65 \%$ of patients (17). The imaging features of FCD can range from minor gray-white junction blurring and subcortical signal abnormalities to evident cortical malformation (18). Furthermore, the examination of MRI and EcoG may be critical in determining the approach to resect lesions. Following surgery for epilepsy, the EEG revealed that epileptiform waves were significantly reduced (19). Moreover, the anxiety of patients was gradually relieved and the quality of life was evidently improved.

The aims of the present study were: i) To assess the outcome of adult patients with FCD IIa after surgery for epilepsy; ii) to investigate the clinical characteristics, EEG and MRI changes and quality of life in adults with FCD IIa after surgery for epilepsy; and iii) to evaluate the prognostic factors influencing the outcome of epilepsy.

\section{Materials and methods}

Patient selection and presurgical evaluation. A total of 110 patients were assessed between 2007 and 2015, and those with a definite pathological diagnosis of FCD IIa were included in the present study. The data from epilepsy surgeries at the Second Affiliated Hospital of Dalian Medical University were analysed retrospectively. Patients with epilepsy were eligible for inclusion in the study if they were $\geq 16$ years old. All surgical patients who participated in the present study represented individuals who had undergone surgery at least one year before completing the study materials. Moreover, all patients gave informed consent for analysis of the clinical data.
The present study was approved by the ethics committee of the Second Affiliated Hospital of Dalian Medical University.

Presurgical evaluation included detailed personal history, date of seizure onset, type of seizure (simple partial, complex partial and/or generalized), seizure frequency, seizure duration, medications and other symptoms (such as the frequency of AEDs). Other preoperative evaluations included EEG, ECoG, high-resolution MRI, neuropsychological assessment and quality of life. Preoperative MRI was independently reviewed by two experienced neuroradiologists. Imaging features included: i) Focal cortical thickening, often accompanied by abnormal cortical sulcation; ii) blurring of the gray-white matter junction; iii) abnormal signal intensity of the cortex on the T2-weighted of fluid-attenuated inversion recovery images iv) transmantle signs; and v) cortical thinning or atrophy. An EEG examination used the Nicolet application where the video electroencephalogram (VEEG, which combines the EEG monitoring system with a video recording device, and can simultaneously record clinical manifestation and EEG of the epilepsy patients) was monitored with a 32/64/128 leads (all capable of recodring different waves) EEG for long-range EEG for an average of 5 days (1-10 days). According to the video data, we can carefully observe the clinical manifestations of these patients. The scalp EEG is according to the standard of 10-20 electrodes placed system records, with special circumstances. For example a sphenoid electrode could be placed in the anterior temporal area. Moreover, the cortex EEG can be placed for parts or figures according to the scalp EEG impression. EEG was read by at least two experienced EEG technicians by independent reading and reporting, and a consensus was reached. The epileptic discharge of the intermittent periods of the seizure was then divided into four categories from VEEG: i) Spike; ii) spine-slow; iii) multiple-spike; and iv) fast rhythm wave. ECoG was routinely performed during all surgeries in the epilepsy surgery program used in the present study. In addition, ECoG provides a unique opportunity to assess the epileptogenicity of exposed cortical areas during surgery. This technique is based on the assumption that epileptiform discharges recorded from the cortex are indicators of local cortical involvement in an epileptogenic network. After opening the dura mater, an effective number of 16 electrodes with intercontact distances of $10 \mathrm{~mm}$ were placed on the exposed cortex surface. However, the electrodes were usually repositioned during the procedure resulting in a larger sampling area.

Surgery and postoperative outcome. There were patients who underwent complete or partial lesionectomy. The type of surgery performed was based on the results of the preoperative evaluation. The surgical area was then decided based on the clinical, neuroimaging and electrophysiological results. After a one-year follow-up, a postoperative MRI and scalp EEG were performed. The outcomes of the seizures were obtained by telephone interviews and from the patients' medical charts, and the surgical outcome was classified according to Engel's four-category classification (20). Class I, free of disabling seizures or auras only; class II, rare seizures ( $\leq$ two seizures/year or $\geq 90 \%$ seizure reduction); class III, worthwhile improvement reduction of seizure frequency $\geq 75 \%$; and class IV, no worthwhile improvement, reduction of seizure 
frequency $<75 \%$. Class I is free of seizures and classes II-IV represent poorer control of seizures following surgical treatment.

Health-related quality of life was evaluated using the quality of life in epilepsy (QOLIE) scale (21) before and at one year after the surgery. A 10-item questionnaire (QOLIE-10) was used for screening patients with epilepsy about the impact of the epilepsy on their lives. It entails a subset of items from the QOLIE-89 questionnaire (22). The total QOLIE-10 scores range between 5 and 50, where high scores indicate a poor quality of life. Moreover, the QOLIE-10 was brief, simple and easy to be completed, therefore, it was selected as a measure of health-related quality of life.

Statistical analysis. An independent sample t-test and $\chi^{2}$-test were conducted to compare the favorable (Engel class I) and the poor (Engel class II-IV) outcomes with the following criteria: i) Gender and age at epilepsy onset, age at epilepsy surgery, duration of epilepsy, seizure frequency, location of resection, side of the brain lesion, past medical history, medicine history, anti-epileptic drug (AED) numbers and MRI; ii) cases with or without EcoG; and iii) complete resection or none. A paired-sample t-test was used to study the changes of EEG before and one year after the surgery. In addition, a multivariate analysis of seizure recurrence was conducted using Cox proportional. The probability of remaining free of disabling seizures was calculated using Kaplan-Meier survival analysis, and all of the analyses were conducted using SPSS version 20.0 (IBM SPSS, Armonk, NY, USA). P<0.05 was used to indicate a statistically significant difference.

\section{Results}

Baseline characteristics. The baseline characteristics for the study samples are presented in Table I. Among the 110 adult patients ( $\geq 16$ years old) there were 52 males and 58 females. The age of the onset of epilepsy ranged between 1 and 53 years old (mean age, 14.06 years). Furthermore, the age of the surgery for epilepsy ranged between 16 and 69 years old (mean age, 30.92 years). The mean duration of epilepsy before surgery was 16.86 years (range, $1-44$ years). In addition, the mean duration of their follow-up was 54.18 months (range, 12-84 months). Past medical history existed in 37 cases $(33.6 \%)$, meanwhile 16 cases had febrile convulsion, 10 cases had hypoxia and 11 cases had encephalitis history. The lesions were resected completely in 96 cases $(87.3 \%)$ and were resected incompletely in 14 cases $(12.7 \%)$. Moreover, MRI was performed in all of the patients. In total, 68/110 patients $(61.8 \%)$ demonstrated visible abnormalities on the MRI, whereas the remaining 42 patients (38.2\%) had no abnormalities observed on an MRI. The lesions were located on the left side in 64 cases and on the right side in 46 cases. Out of all the patients, 78 (70.9\%) had temporal lobe epilepsy, 15 (13.6\%) had frontal lobe epilepsy and $3(2.7 \%)$ had parietal lobe epilepsy, and were referred for surgical consideration following several therapeutic attempts with different AEDs (range, 0-7) and with poor results on their seizures.

Histopathological features of FCD IIa. Histopathological features of FCD IIa can be diagnosed by H\&E staining and
Table I. Baseline characteristics of the 110 enrolled patients.

\begin{tabular}{lc}
\hline Characteristic & Value \\
\hline Patients included, $\mathrm{n}$ & 110 \\
Male $^{\mathrm{a}}$ & $52(47.3)$ \\
Age at epilepsy onset, years $^{\mathrm{b}}$ & $14.06(10.62)$ \\
Age at epilepsy surgery, years $^{\mathrm{b}}$ & $30.92(11.13)$ \\
Mean duration of epilepsy, years $^{\mathrm{b}}$ & $16.86(9.74)$ \\
Past medical history $^{\mathrm{a}}$ & $37(33.6)$ \\
Complete resection $^{\mathrm{a}}$ & $96(87.3)$ \\
Left side of lesions $^{\mathrm{a}}$ & $64(58.2)$ \\
AED category $^{\mathrm{b}}$ & $2.39(1.26)$ \\
Location of resection $^{\mathrm{a}}$ & \\
Temporal lobe & $78(70.9)$ \\
Extratemporal lobe & $23(20.9)$ \\
Multilobe lesionectomy $^{\text {Follow up, months }}{ }^{\mathrm{b}}$ & $9(8.2)$ \\
\end{tabular}

Data are presented as ${ }^{\mathrm{a}} \mathrm{N}(\%),{ }^{\mathrm{b}}$ mean (standard deviation); AED, anti-epileptic drug.

immunohistochemistry (Fig. 1). All the cases mentioned in the present study were diagnosed by two pathologists. $H \& E$ staining, revealed that the structure of neurons on the dysplastic temporal lobe cortex were arranged in disorder. Moreover, architectural abnormalities with giant cells and dysmorphic neurons but no balloon cells are shown in Fig. 1A. Gliosis was often observed in the brain tissues from patients with refractory temporal lobe epilepsy together with neuronophagia and neuronal degeneration. In dysmorphic neurons, Nissl substance was aggregated and displaced towards the cell membrane (Fig. 1B). Furthermore, immunohistochemical examination revealed that NeuN displayed a disordered arrangement of neurons (Fig. 1D), GFAP(+) and Olig-2(+) to the glial cells (Fig. 1C), as well as CD34(+) to the vascular endothelium. Finally, all of them underwent focal cortical dysplasia (FCD IIa).

Summary of a seizure-related profile and surgical outcome. For the 110 patients who were followed up for one year after the last epilepsy surgery, 72 patients $(65.4 \%)$ achieved Engel class I, 20 (18.2\%) Engel class II, 11 (10\%) Engel class III, and $7(6.4 \%)$ Engel class IV. Furthermore, the Engel seizure outcome was relevant with the extent of resection $(\mathrm{P}=0.028)$, presurgical MRI $(\mathrm{P}=0.023)$ and presurgical ECoG $(\mathrm{P}=0.001)$. A complete surgical resection and preoperative imaging evaluation in the patients may lead to a positive operation efficacy, whereas no other statistically significant differences were observed between the two Engel class groups with other clinical characteristics, including gender, age at epilepsy onset, duration of epilepsy, age at surgery, the number of AEDs, the patient history, presurgical image or the side and location of surgery. The seizure-related profile and outcomes of the 30 patients are summarized in Table II.

ECoG is different from scalp EEG. EcoG is an accurate and stable location method, particularly when the results of the 

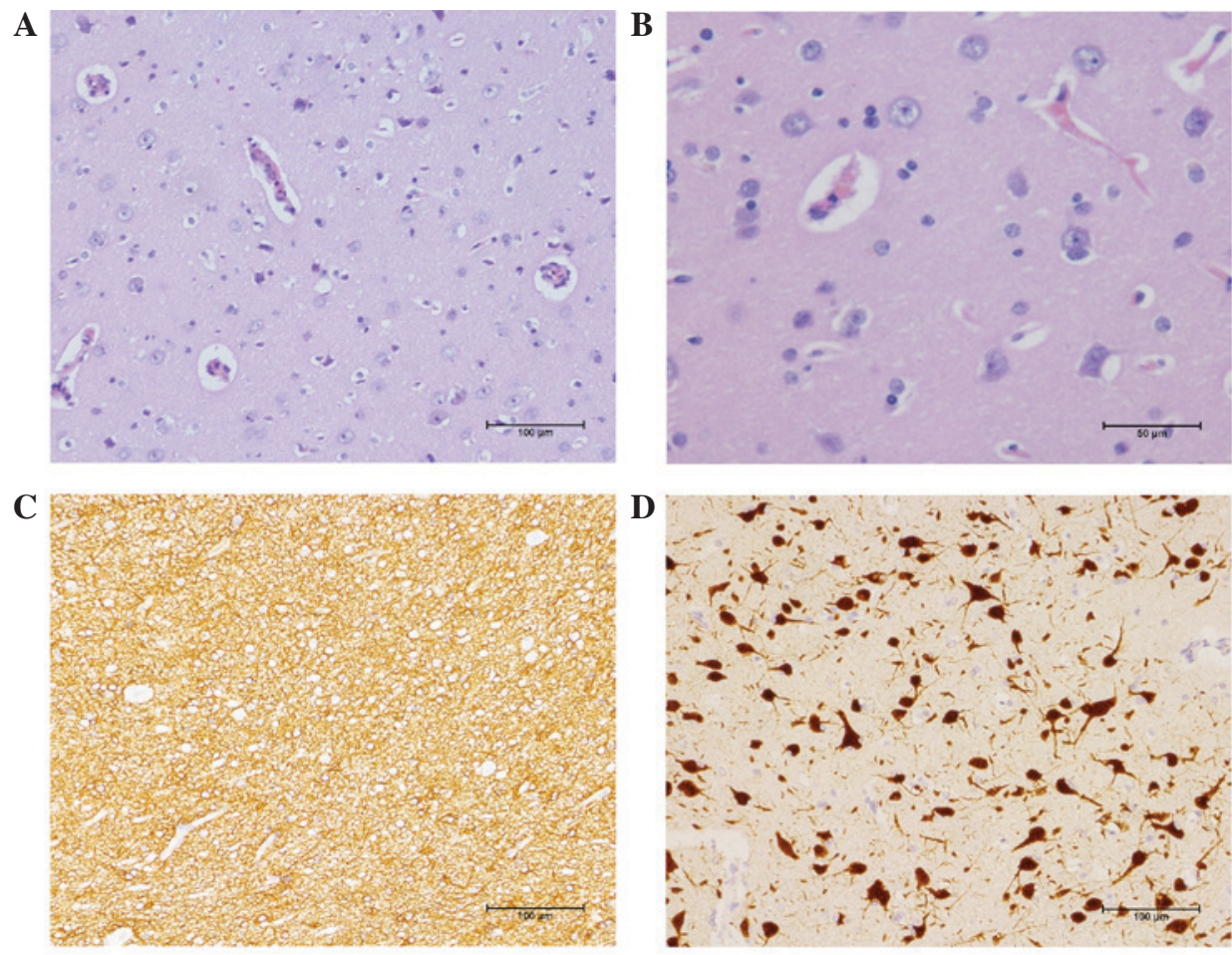

Figure 1. Histopathological features of FCD IIa. (A) Dysmorphic neurons with nuclear, cytoplasmic, and axonal atypia (H\&E; magnification, x200). (B) High-power magnification of dysmorphic neurons. In the dysmorphic neurons, Nissl substance is aggregated and displaced towards the cell membrane (H\&E; magnification, x400). (C) GFAP(+), GFAP gliosis was observed to different extent. (D) NeuN(+), disordered arrangement of neurons. H\&E, haematoxylin and eosin; GFAP, glial fibrillary acidic protein.

MRI are negative. Therefore, in order to resect chronic lesions completely, preoperative ECoG was required. In the current study when $>5$ seizures were identified and there were more than three times from epileptic focus. Then the neurosurgeon was able to determine the range of the precise epilepsy lesion according to ECoG monitoring (Fig. 2A and B). Intraoperative ECoG monitoring is also indispensable in patients who have undergone surgery for epilepsy. The neurosurgeon should be based on MRI lesions or ECoG accurate location of lesions to guide cortex electrode plates which could be detected the resection of lesions within the scope of application of each interval to $>10 \mathrm{~mm}$ one electrode to confirm the discharge of seizures (Fig. 2C). At the same time they should determine the scope of a wider area to the surrounding stretch further out if there is still an epileptic focus (Fig. 2D). The discharge epileptogenic zone should be integrated, and triggering nerve dysfunction should be avoided. Furthermore, assessments of ECoG patterns are relevant in order to determine the extent of the resection that can influence the surgery outcome $(\mathrm{P}<0.001)$. Therefore, ECoG monitoring for removal of epilepsy lesions prior to discharge of seizure is significant for the prognosis for surgery.

Multivariate analysis of variables affecting seizure recurrence. For all patients, the median overall seizure freedom was 25.92 months at the time of the last follow-up, and 69/110 cases had experienced a seizure after the surgery. The probability of patients remaining seizure-free was $68 \%$ at one year, $40 \%$ at three years post-surgery and $28 \%$ at five years. Multivariate regression analysis of the clinical characteristics and seizure recurrence are shown in Table III. In the multivariate analysis, the one year seizure recurrence rate was $32 \%$ and three-year seizure recurrence rate was $60 \%$. Furthermore, the statistically significant variables affecting seizure recurrence were preoperative ECoG (HR; 1.929 95\% CI: 1.185-3.128, $\mathrm{P}=0.008$ ) and the extent of resection [(HR; $2.56595 \%$ CI:1.331-4.943, $\mathrm{P}=0.005)$ ], preoperative $\mathrm{ECoG}$ and complete resection of lesions could significantly reduce the seizure recurrence rate. Meanwhile, the location of resection and preoperative seizure frequency can also influence the seizure recurrence. The seizure recurrence rate of patients with temporal lobe epilepsy was evidently reduced compared to those with extratemporal lobe epilepsy and multilobe lesionectomy. Furthermore, the patients with preoperative seizure frequency $<10$ per month could result in a better prognosis. By contrast, no statistically significant correlation with seizure recurrence and gender, age at epilepsy onset, duration of epilepsy, age at surgery, the number of AEDs, the history of the patient and the presurgical image or the side of surgery was identified $(\mathrm{P}>0.05)$, and they were also not included in Table III.

Among all patients, the one-year seizure-free rate was 82 and 53\%, and the three-year rates were 47 and 33\% in patients with or without preoperative ECoG (Fig. 3A). Furthermore, the log-rank test and Kaplan-Meier method indicated that preoperative ECoG could significantly enhance the seizure-free rate $(\mathrm{P}=0.009)$. The median seizure-free period was 45 months in the temporal, 19 months in the extratemporal and 17 months in the multilobe epilepsy groups, which indicated that the prognosis of the temporal epilepsy group was evidently better than the other two groups ( $\mathrm{P}=0.012)$ (Fig. 3B). Meanwhile, the log-rank test and Kaplan-Meier method indicated a significantly poor survival in patients with incomplete 
Table II. Summary of seizure-related profile and surgical outcome of the 110 patients.

\begin{tabular}{|c|c|c|c|c|}
\hline \multirow[b]{2}{*}{ Characteristics } & \multicolumn{2}{|c|}{ Engel class } & \multirow[b]{2}{*}{ Statistics } & \multirow[b]{2}{*}{ P-value } \\
\hline & I & II-IV & & \\
\hline Age at epilepsy surgery (years) & 30.85 & 31.05 & $\mathrm{t}=-0.092$ & 0.927 \\
\hline Age at epilepsy onset (years) & 13.99 & 14.18 & $\mathrm{t}=-0.089$ & 0.929 \\
\hline Duration of epilepsy (years) & 16.85 & 16.87 & $\mathrm{t}=-0.007$ & 0.994 \\
\hline AED category & 2.4 & 2.37 & $\mathrm{t}=0.135$ & 0.893 \\
\hline Gender & & & & 0.677 \\
\hline Male & 33 & 19 & $\mathrm{x}^{2}=0.173$ & \\
\hline Female & 39 & 19 & & \\
\hline Extent of resection & & & & 0.028 \\
\hline Complete resection & 67 & 29 & $\mathrm{x}^{2}=4.858$ & \\
\hline Incomplete resection & 5 & 9 & & \\
\hline Past medical history & & & & 0.926 \\
\hline Yes & 24 & 13 & $x^{2}=0.009$ & \\
\hline No & 48 & 25 & & \\
\hline Side of lesion & & & & 0.652 \\
\hline Left & 43 & 21 & $x^{2}=0.203$ & \\
\hline Right & 29 & 17 & & \\
\hline Location of resection & & & & 0.597 \\
\hline Temporal lobe & 53 & 25 & $\mathrm{x}^{2}=1.032$ & \\
\hline Extratemporal lobe & 13 & 10 & & \\
\hline Multilobe lesionectomy & 6 & 3 & & \\
\hline Presurgical MRI & & & & 0.023 \\
\hline+ & 50 & 18 & $x^{2}=5.136$ & \\
\hline- & 22 & 20 & & \\
\hline Presurgical ECoG & & & & 0.001 \\
\hline Yes & 45 & 11 & $x^{2}=11.204$ & \\
\hline No & 27 & 27 & & \\
\hline
\end{tabular}

AED, anti-epileptic drug; MRI, magnetic resonance imaging; ECoG, electrocorticography.

resection of the lesion $(\mathrm{P}=0.005)$ and higher preoperative seizure frequency $(\mathrm{P}=0.033)$ (Fig. 3C and D).

Quality of life in epileptic patients. Items 1-10 of the QOLIE-10 included epilepsy-related effects (memory, physical effects, mental effects of medication), mental health (energy, depression, overall quality of life) and role functioning (seizure worry, work, driving, social limitations). The preoperative and postoperative scores of the ten items are shown in Fig. 4. Furthermore, the total mean scores of the 110 patients were $29.3 \pm 2.74$ and $18.5 \pm 4.55$ before and at one year after the surgery for epilepsy, respectively, and the difference was shown to be statistically significant $(\mathrm{P}<0.01)$.

\section{Discussion}

FCD is caused by neuronal proliferation and differentiation, cortical functional architecture and migration anomalies that were firstly described by Taylor et al in 1971 (1). FCD has increasingly been diagnosed as a cause of symptomatic epilepsy, particularly for refractory epilepsy in paediatry and as the second or third common cause of adult patients who have undergone surgery for epilepsy (23). It has been reported that the incidence of epilepsy was $\sim 46.5 \%$ (15\% in adults) and (25\% in children) (24). These percentages are significantly higher than the $1.7 \%$ observed in the general population (24). Furthermore, the incidence of FCD was higher but its etiology, clinical features, imaging and pathological features were not fully understood, which often lead to delayed diagnosis (25). Due to the severity and refractoriness to drug therapy, surgical treatment of epilepsy associated with FCD could be a valid therapeutic option (26). Furthermore surgical specimens allow for a precise neuropathological diagnosis and identification of histopathological variants (27). Though the operation faces great change and innovation, the seizure remission rate of the epilepsy surgery increases continuously (28), reaching $50-70 \%$ according to numerous clinical case reports $(29,30)$. The abnormal MRI signals and EEG discharges, as well as the range of FCD lesions and its concerned functional area are all possible influence factors that make it difficult to choose 
Table III. Multivariate analysis of risk factors for seizure recurrence.

\begin{tabular}{lcc}
\hline Risk factor & $\mathrm{n}$ & HR $(95 \% \mathrm{CI})$ \\
\hline Preoperative ECoG & & P-value \\
Yes & 56 & $1.925(1.185-3.128)$ \\
No & 54 & Reference \\
Extent of resection & & $2.565(1.331-4.943)$ \\
Complete resection & 96 & Reference \\
Incomplete resection & 14 & $0.391(0.179-0.857)$ \\
Location of resection & & $0.608(0.254-1.458)$ \\
Temporal lobe & 78 & Reference \\
Extratemporal lobe & 23 & $0.488(0.263-0.905)$ \\
Multilobe lesionectomy & 9 & $0.756(0.358-1.592)$ \\
Home seizure-frequency & 68 & 0.019 \\
Low (<10/month) & 25 & 0.265 \\
Medium (10-30/month) & 17 & 0.023 \\
High (>30/month) & & 0.461
\end{tabular}

HR, hazard ratio; ECoG, electrocorticography; CI, confidence interval.

A

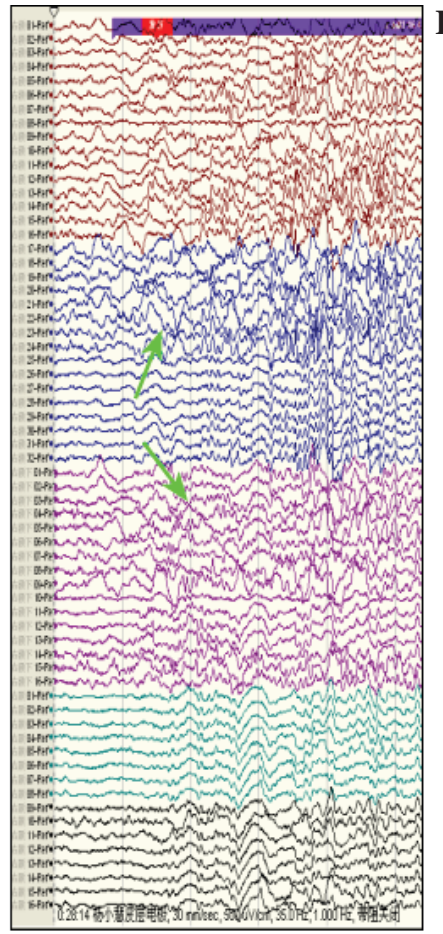

B

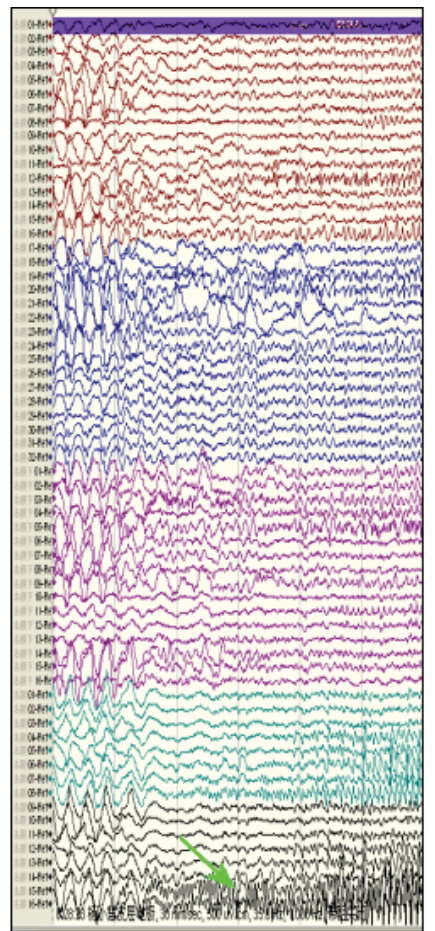

$\mathrm{C}$
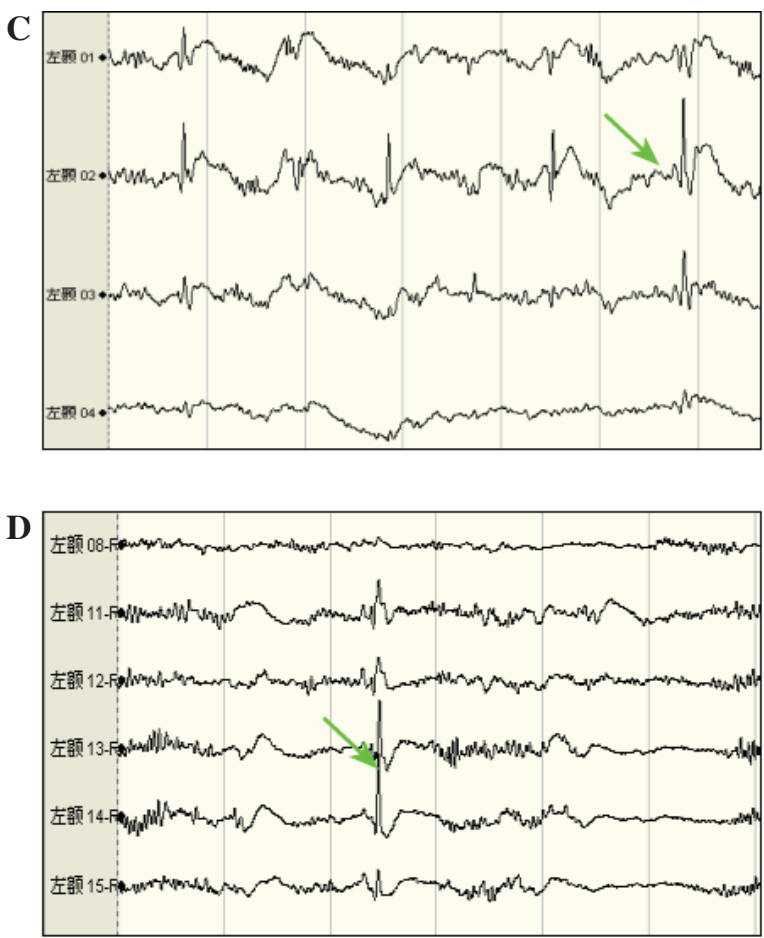

Figure 2. Electrocorticography recordings in 3 patients. (A) Cortex EEG electrodes are placed in the right frontal temporal parts, and the origin area can be seen in the right frontal lobe. (B) The complex partial seizure generated from the right temporal lobe, so the cortex electrode can confirm the epileptogenic zone and the inital discharge area. (C and D) The results of the cortical EEG monitoring facilitates the determination of the accurate resection range.

operation strategies. Compared with other intractable epilepsy surgeries (31), the FCD surgery complication and fatality rates are very low; reportedly $2 \%$ (31). Thus far, surgery treatment has achieved satisfactory results in patients with FCD-derived intractable epilepsy. Through retrospective analysis of epilepsy patients in our centre, the pathology of the majority of the patients was identified as FCD IIa and the prognosis of them were favorable. Fauser et al (32) conducted a retrospective study of 67 patients with FCD, and identified the proportion of patients with Engel I and FCD IIa 12 months after the surgery to be $57.0 \%$. However, the study by Widdess-Walsh et al (33) revealed that the proportion of patients with Engel I was $67 \%$. Hence, a total of 110 patients with a definite pathological diagnosis of FCD IIa were retrospectively analysed, and the proportion of patients with Engel I was found to be $65.4 \%$ which was close to the results of other studies (34). At present, 
A

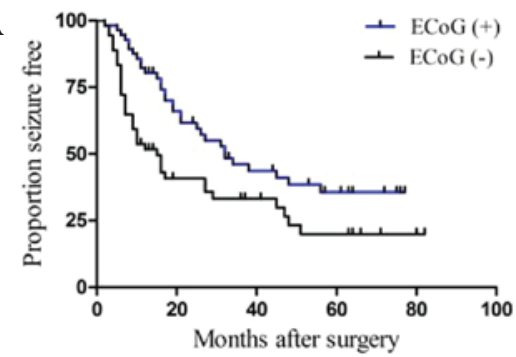

C

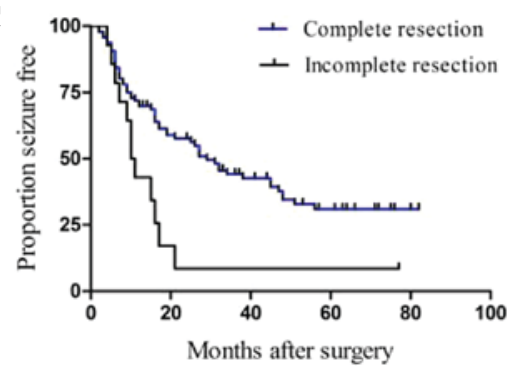

B

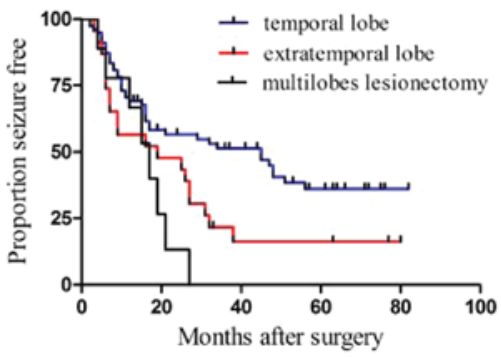

D

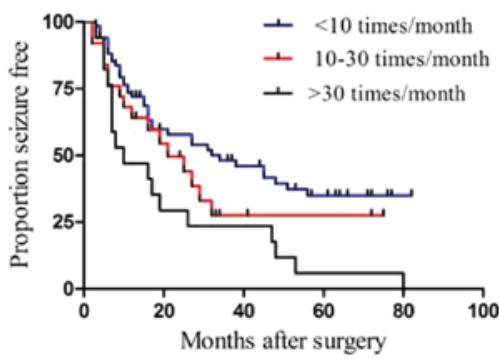

Figure 3. Kaplan-Meier graph of seizure-free rate according to (A) ECoG; (B) location of resection; (C) extent of resection and (D) preoperative seizure frequency. ECoG, electrocorticography.
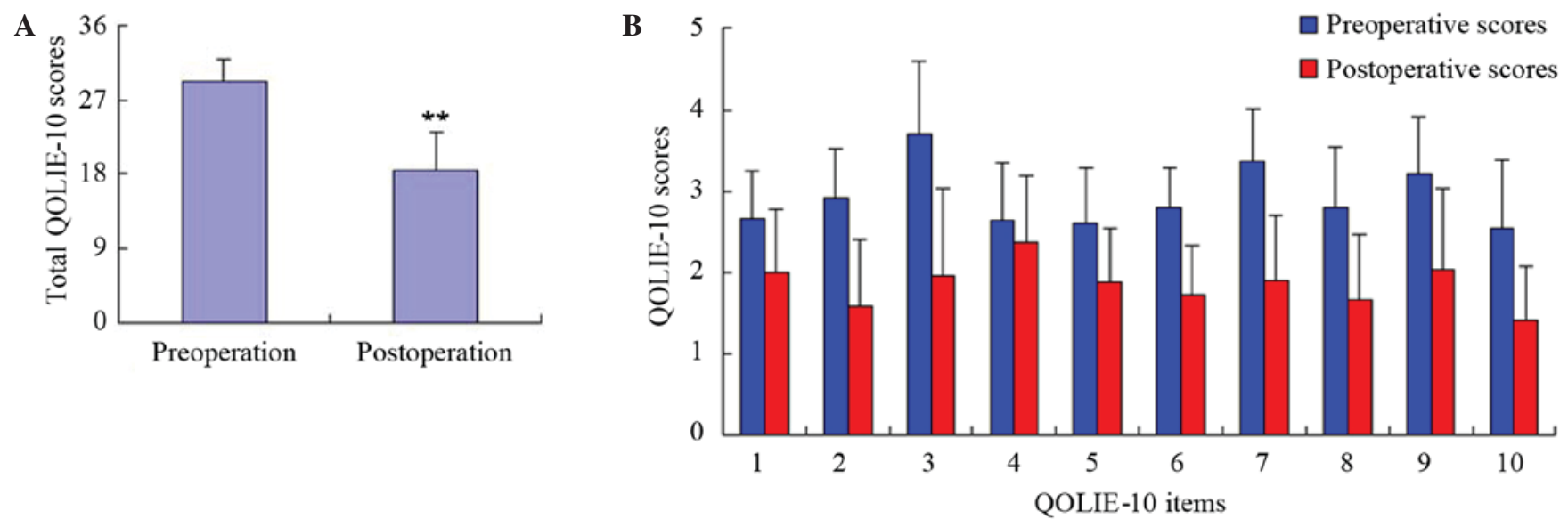

Figure 4. (A) Total scores of the 110 patients before and 1 year after the epilepsy surgery. ${ }^{* *} \mathrm{P}<0.01$ vs. preoperative total scores. (B) Preoperative and postoperative scores of the items of QOLIE-10 before and 1 year after the epilepsy surgery. Data presented as the mean \pm standard deviation, $\mathrm{n}=110$ per item. 1: Energy; 2: Depression; 3: Driving; 4: Memory; 5: Work; 6: Social; 7: Physical effect; 8: Mental effect; 9: Seizure worry; and 10: Overall quality of life. SD, standard deviation; QOLIE, quality of life in epilepsy.

there are numerous studies on refractory epilepsy in children, and the prognosis is satisfactory (35). However, there is a shortage of studies on refractory epilepsy in adults. Therefore, the present study focused on the clinical characteristics and prognosis of adult patients with FCD IIa and provided the theoretical basis for the surgical treatment of epilepsy in the future.

In the present study, clinical characteristics of epilepsy were analysed in 110 adult patients with histologically proven FCD IIa. The correlation between clinical indexes and Engel classes over a one-year follow-up were emphatically analysed in order to explore the clinical factors that influenced the short-term prognosis of epileptic patients with FCD IIa. Out of the 110 patients, $72(65.4 \%)$ achieved Engel class I, 20 (18.2\%) Engel class II, 11 (10\%) Engel class III and 7 (6.4\%) Engel class IV. Engel Class I was defined as the better prognosis group, Engel class II-IV as the poor prognosis group. Through statistical tests, the present study revealed that there were no other statistically significant differences between the Engel class and other clinical indexes, including gender, age at epilepsy onset, duration of epilepsy, age at surgery, seizure frequency, number of AEDs, patient history, presurgical image or the side and location of surgery. Furthermore, a complete resection of the lesion was the most important clinical index that influenced the postoperative Engel class $(\mathrm{P}=0.028)$. Complete resection of FCD, including the surrounding epileptogenic areas, is the most important predictor of seizure outcome $(36,37)$. Furthermore, a complete surgical resection in the patients may lead to a positive operation efficacy, but in patients with an incomplete excision, seizure recurrence has been related to the extension of dysplastic cortex beyond the visible abnormality (38). Lerner et al (39) reviewed recent literature regarding the surgical treatment of FCD, and revealed that $65-70 \%$ of the patients can achieve a complete 
resection. Furthermore, the complete remission rate of the seizure in patients with complete resection of the lesions was $\sim 77 \%$, and $20 \%$ in patients with an incomplete resection. Rowland et al (40) conducted a meta-analysis, and demonstrated that the most significant prognostic factor for surgical treatment of FCD was whether the lesions were resected completely. A complete resection of FCD can influence the short-term outcome of epilepsy, however, a multimodality approach and teamwork are required in order to achieve this goal. Furthermore, a clear localization of the epileptic focus is required, and MRI and EcoG examination were important for this.

Functional MRI is a non-invasive method used for evaluation of locating the epileptic lesions preoperatively. The characteristics of MRI results in patients with FCD IIa typically include a gray-white junction blurring, cortex thickening, abnormal outer and abnormal signal of the cortex and white matter in T2/Flair. MRI has important diagnostic value for FCD; however, there remain cases of FCDs that are difficult to identify using MRI. Literature reported that the diagnostic rate of FCD by MRI was 60-90\% (41). Widdess-Walsh et al (33) analysed 40 patients with FCD-II and revealed that the positive rate of MR was $75 \%$ in patients with FCD IIA whereas Krsek et al (29) demonstrated that the positive rate of MR was $78 \%$ in patients with FCD IIA. Moreover, previous studies have indicated that patients with a positive MRI had a higher remission rate of surgical treatment (42). The present study revealed that the positive rate of MR was $61.8 \%$ in 110 adult patients with FCD IIa, and that there was a significant correlation between the positive rate of $\mathrm{MR}$ and Engel class one year following operation $(\mathrm{P}=0.023)$. To date, there are numerous reports on discharge characteristics of scalp EEG in patients with FCD (43). Preoperative and intraoperative ECoG was routinely performed during all surgeries in our epilepsy surgery program. ECoG provides a unique opportunity to assess the epileptogenicity of exposed cortical areas. Currently, 80-84\% of epilepsy centers worldwide use EcoG to guide intraoperative surgical removal (44). ECoG is different from scalp EEG, it is an accurate and stable location method, particularly when the results of the MRI are negative. In order to completely resect, ECoG monitoring was necessary. The applications of EcoG in epilepsy operation are mainly concentrated in the following aspects: i) Long-range cortical electrode monitoring in preoperative assessment is used in those cases where there has been a difficulty for negative MRI and scalp EEG epileptogenic zone localization; ii) intraoperative ECoG could determine the position and range of the epileptogenic focus, and guide the surgical strategy; iii) after removing a lesion, reiteration EcoG results could guide further treatment and evaluation of prognosis; and iv) intraoperative cortical electrical stimulation could locate functional areas as far as possible to avoid nerve dysfunction. However, intraoperative EcoG demonstrates period electrical activity of the intermittent periods. Nevertheless, the origin of the epileptic area remains controversial. The present study revealed that $50.9 \%$ of patients performed a preoperative EcoG in 110 patients with FCDIIA. Moreover, EcoG positioning was significantly related with postoperative Engel classification at one year after surgery $(\mathrm{P}=0.001)$.
The present study aimed to investigate the prognostic factors and the risk of seizure recurrence following surgery for epilepsy. Furthermore, the risk associated with preoperative and operative factors was assessed. In the analysis of the present study the occurrence of any disabling postoperative seizure was assessed as a recurrence. In common with a number of other studies $(45,46)$, multivariate regression analyses with forward elimination revealed that in epileptic patients with FCD IIa the extent of resection, seizure frequency, preoperative ECoG and location of resection were the most important factors that significantly increased the risk of not becoming seizure-free postoperatively. By contrast, the gender, age at epilepsy onset, duration of epilepsy, age at surgery, number of AEDs, patient history, presurgical image and the side of surgery were not significant prognostic factors for the postoperative epilepsy outcome. Epilepsy is a chronic disorder, characterized by unpredictable, uncontrolled seizures that interfere with patient lifestyles, activities and interests. The condition introduces a number of psychosocial challenges and adaptive demands, and threatens the quality of life. Recently, the medical community pays increasingly more attention to quality of life improvement and the psychological state in patients with epilepsy (47). Moreover, the evaluation of the quality of life has gradually become an important index of clinical therapeutic effects (48). The QOLIE-10 scale is the current internationally recognized method for evaluating the quality of life in patients with epilepsy. The present study revealed that problems with the quality of life in patients with FCD, and in the majority of cases experience panic (49). Therefore more attention should be paid on the mental health problems in patients with epilepsy in order to prevent patients from giving up their lives. In addition, the preoperative and postoperative life quality in patients with FCD changed significantly. The postoperative quality of life and epileptic remission rate were evidently improved $(\mathrm{P}=0.000)$, which indicated that epilepsy surgery was important in the treatment of refractory epilepsy. However, if we aimed to achieve more ideal surgical outcomes, a series of neuroimaging examinations before the operation would be required. In this way, improved surgical strategies and improved remission rates of postoperative epilepsy could be developed. Using the QOLIE-10 scale to assess the quality of life in patients with FCD can provide an objective basis for improving the cure rates of epilepsy surgery.

For refractory epilepsy with FCD IIa, earlier surgical intervention should be indicated. Complete resection is the single most important index influencing the Engel class at one year after surgery, and requires a multimodality approach and teamwork in order to achieve this goal. MRI and EcoG can help locate epileptogenic foci and improve the patients' postoperative epileptic remission rate. A multivariate analysis was conducted using Cox proportional hazard regression to assess the long-term prognosis of epilepsy caused by FCD IIa, and found that preoperative ECoG, the extent of resection, location of resection and preoperative seizure frequency were important prognostic factors for seizures following surgery. Furthermore, surgical intervention and complete resection of the lesion helps improve seizure control, physical and mental health and quality of life. Despite the difficulties associated with a complete resection, the majority of the patients achieve 
a notable reduction in seizures. Therefore, this data may be helpful when counseling patients before undergoing surgery.

\section{Acknowledgements}

The authors thank Mr. Hang Yin for his effort in collecting and organizing literature and data. We also thank the physicians who referred the patients to epilepsy surgery.

\section{References}

1. Taylor DC, Falconer MA, Bruton CJ and Corsellis JA: Foca dysplasia of the cerebral cortex in epilepsy. J Neurol Neurosurg Psychiatry 34: 369-387, 1971.

2. Guerrini R, Dobyns WB and Barkovich AJ: Abnormal development of the human cerebral cortex: Genetics, functional consequences and treatment options. Trends Neurosci 31: 154-162, 2008.

3. Fauser S, Essang C, Altenmüller DM, Staack AM, Steinhoff BJ, Strobl K, Bast T, Schubert-Bast S, Stephani U, Wiegand G, et al: Long-term seizure outcome in 211 patients with focal cortical dysplasia. Epilepsia 56: 66-76, 2015.

4. Prayson RA: Classification and pathological characteristics of the cortical dysplasias. Childs Nerv Syst 30: 1805-1812, 2014

5. Blümcke I, Thom M, Aronica E, Armstrong DD, Vinters HV, Palmini A, Jacques TS, Avanzini G, Barkovich AJ, Battaglia G, et al: The clinicopathological spectrum of focal cortical dysplasias: A consensus classification proposed by an ad hoc task force of the ILAE diagnostic methods commission. Epilepsia 52: 158-174, 2011.

6. Raymond AA, Fish DR, Sisodiya SM, Alsanjari N, Stevens JM and Shorvon SD: Abnormalities of gyration, heterotopias, tuberous sclerosis, focal cortical dysplasia, microdysgenesis, dysembryoplastic neuroepithelial tumour and dysgenesis of the archicortex in epilepsy. Clinical, EEG and neuroimaging features in 100 adult patients. Brain 118: 629-660, 1995.

7. Keene DL, Jimenez CC and Ventureyra E: Cortical microdysplasia and surgical outcome in refractory epilepsy of childhood. Pediatr Neurosurg 29: 69-72, 1998.

8. Cohen-Gadol AA, Ozduman K, Bronen RA, Kim JH and Spencer DD: Long-term outcome after epilepsy surgery for focal cortical dysplasia. J Neurosurg 101: 55-65, 2004.

9. Kral T, Clusmann H, Blümcke I, Fimmers R, Ostertun B, Kurthen $\mathrm{M}$ and Schramm J: Outcome of epilepsy surgery in focal cortical dysplasia. J Neurol Neurosurg Psychiatry 74: $183-188,2003$.

10. Asadi-Pooya AA, Nei M, Sharan A and Sperling MR: Patient Historical Risk Factors Associated with Seizure Outcome After Surgery for Drug-Resistant Nonlesional Temporal Lobe Epilepsy. World Neurosurg 91: 205-209, 2016.

11. Perry MS, Dunoyer C, Dean P, Bhatia S, Bavariya A, Ragheb J, Miller I, Resnick T, Jayakar P and Duchowny M: Predictors of seizure freedom after incomplete resection in children. Neurology 75: 1448-1453, 2010.

12. Rowland NC, Englot DJ, Cage TA, Sughrue ME, Barbaro NM and Chang EF: A meta-analysis of predictors of seizure freedom in the surgical management of focal cortical dysplasia. J Neurosurg 116: 1035-1041, 2012.

13. Yun CH, Lee SK, Lee SY, Kim KK, Jeong SW and Chung CK: Prognostic factors in neocortical epilepsy surgery: Multivariate analysis. Epilepsia 47: 574-579, 2006.

14. Wennberg R, Quesney LF, Lozano A, Olivier A and Rasmussen T: Role of electrocorticography at surgery for lesion-related frontal lobe epilepsy. Can J Neurol Sci 26: 33-39, 1999.

15. Chang EF, Wang DD, Barkovich AJ, Tihan T, Auguste KI, Sullivan JE, Garcia PA and Barbaro NM: Predictors of seizure freedom after surgery for malformations of cortical development. Ann Neurol 70: 151-162, 2011.

16. Kim YH, Kang HC, Kim DS, Kim SH, Shim KW, Kim HD and Lee JS: Neuroimaging in identifying focal cortical dysplasia and prognostic factors in pediatric and adolescent epilepsy surgery. Epilepsia 52: 722-727, 2011.

17. Hauptman JS and Mathern GW: Surgical treatment of epilepsy associated with cortical dysplasia: 2012 update. Epilepsia 53 (Suppl 4): S98-S104, 2012.
18. Colon AJ, van Osch MJ, Buijs M, Grond JV, Boon P, van Buchem MA and Hofman PA: Detection superiority of 7 T MRI protocol in patients with epilepsy and suspected focal cortical dysplasia. Acta Neurol Belg 116: 259-269, 2016.

19. Alshafai L, Ochi A, Go C, McCoy B, Hawkins C, Otsubo H, Snead OC, Rutka J and Widjaja E: Clinical, EEG, MRI, MEG, and surgical outcomes of pediatric epilepsy with astrocytic inclusions versus focal cortical dysplasia. Epilepsia 55: 1568-1575, 2014.

20. Engel J Jr (Ed): Outcome with respect to epileptic seizures. In: Surgical treatment of the epilepsies. 2nd edition. Raven Press, pp609-621, 1993.

21. Cramer JA, Perrine K, Devinsky O and Meador K: A brief questionnaire screen for quality of life in epilepsy: The QOLIE-10. Epilepsia 37: 577-582, 1996.

22. Devinsky O, Vickrey BG, Cramer J, Perrine K, Hermann B, Meador K and Hays RD: Development of the quality of life in epilepsy inventory. Epilepsia 36: 1089-1104, 1995.

23. Becker AJ, Blümcke I, Urbach H, Hans V and Majores M: Molecular neuropathology of epilepsy-associated glioneuronal malformations. J Neuropathol Exp Neurol 65: 99-108, 2006.

24. Bast T, Ramantani G, Seitz A and Rating D: Focal cortical dysplasia: Prevalence, clinical presentation and epilepsy in children and adults. Acta Neurol Scand 113: 72-81, 2006.

25. Guerrini R, Duchowny M, Jayakar P, Krsek P, Kahane P, Tassi L, Melani F, Polster T, Andre VM, Cepeda C, et al: Diagnostic methods and treatment options for focal cortical dysplasia. Epilepsia 56: 1669-1686, 2015.

26. Kwon HE, Eom S, Kang HC, Lee JS, Kim SH, Kim DS and Kim HD: Surgical treatment of pediatric focal cortical dysplasia: Clinical spectrum and surgical outcome. Neurology 87: 945-951, 2016.

27. Tassi L, Garbelli R, Colombo N, Bramerio M, Lo Russo G, Deleo F, Milesi G and Spreafico R: Type I focal cortical dysplasia: Surgical outcome is related to histopathology. Epileptic Disord 12: 181-191, 2010.

28. Hemb M, Velasco TR, Parnes MS, Wu JY, Lerner JT, Matsumoto JH, Yudovin S, Shields WD, Sankar R, Salamon N, Vinters HV and Mathern GW: Improved outcomes in pediatric epilepsy surgery: The UCLA experience, 1986-2008. Neurology 74: 1768-1775, 2010.

29. Krsek P, Pieper T, Karlmeier A, Hildebrandt M, Kolodziejczyk D, Winkler P, Pauli E, Blümcke I and Holthausen H: Different presurgical characteristics and seizure outcomes in children with focal cortcal dysplasia type I or II. Epilepsia 50: 125-137, 2009.

30. Kim DW, Lee SK, Chu K, Park KI, Lee SY, Lee CH, Chung CK, Choe G and Kim JY: Predictors of surgical outcome and pathologic considerations in focal cortical dysplasia. Neurology 72: 211-216, 2009.

31. Hirfanoglu T, Serdaroglu A, Kurt G, Erdem A, Capraz I, Bilir E, Vural O, Ucar M, Oner AY, Onal B, et al: Outcomes of resective surgery in children and adolescents with focal lesional epilepsy: The experience of a tertiary epilepsy center. Epilepsy Behav 63: 67-72, 2016.

32. Fauser S, Schulze-Bonhage A, Honegger J, Carmona $H$, Huppertz HJ, Pantazis G, Rona S, Bast T, Strobl K, Steinhoff BJ, et al: Focal cortical dysplasias: Surgical outcome in 67 patients in relation to histological subtypes and dual pathology. Brain 127: 2406-2418, 2004.

33. Widdess-Walsh P, Kellinghaus C, Jeha L, Kotagal P, Prayson R, Bingaman W and Najm IM: Electro-clinical and imaging characteristic of focal cortical dysplasia: Correlation with pathological subtypes. Epilepsy Res 67: 25-33, 2005.

34. Widjaja E, Otsubo H, Raybaud C, Ochi A, Chan D, Rutka JT, Snead OC III, Halliday W, Sakuta R, Galicia E, et al: Characteristics of MEG and MRI between Taylor's focal cortical dysplasia (type II) and other cortical dysplasia: Surgical outcome after complete resection of MEG spike source and MR lesion in pediatric cortical dysplasia. Epilepsy Res 82: 147-155, 2008.

35. Sacino MF, Ho CY, Whitehead MT, Zelleke T, Magge SN, Myseros J, Keating RF, Gaillard WD and Oluigbo CO: Resective surgery for focal cortical dysplasia in children: A comparative analysis of the utility of intraoperative magnetic resonance imaging (iMRI). Childs Nerv Syst 32: 1101-1107, 2016.

36. Hader WJ, Mackay M, Otsubo H, Chitoku S, Weiss S, Becker L, Snead OC III and Rutka JT: Cortical dysplastic lesions in children with intractable epilepsy: Role of complete resection. J Neurosurg 100 (2 Suppl Pediatrics): S110-S117, 2004.

37. Phi JH, Cho BK, Wang KC, Lee JY, Hwang YS, Kim KJ, Chae JH, Kim IO, Park SH and Kim SK: Longitudinal analyses of the surgical outcomes of pediatric epilepsy patients with focal cortical dysplasia. J Neurosurg Pediatr 6: 49-56, 2010. 
38. Edwards JC, Wyllie E, Ruggeri PM, Bingaman W, Lüders H, Kotagal P, Dinner DS, Morris HH, Prayson RA and Comair YG: Seizure outcome after surgery for epilepsy due to malformation of cortical development. Neurology 55: 1110-1114, 2000.

39. Lerner JT, Salamon N, Hauptman JS, Velasco TR, Hemb M, Wu JY, Sankar R, Donald Shields W, Engel J Jr, Fried I, et al: Assessment and surgical outcomes for mild type I and severe type II cortical dysplasia: A critical review and the UCLA experience. Epilepsia 50: 1310-1335, 2009.

40. Rowland NC, Englot DJ, Cage TA, Sughrue ME, Barbaro NM and Chang EF: A meta-analysis of predictors of seizure freedom in the surgical management of focal cortical dysplasia. J Neurosurg 116: 1035-1041, 2012.

41. Colombo N, Tassi L, Galii C, Citterio A, Lo Russo G, Scialfa G and Spreafico R: Focal cortical dysplasias: MR imaging, histopathologic, and clinical correlations in surgically treated patients with epilepsy. AJNR Am J Neuroradiol 24: 724-733, 2003.

42. McIntosh AM, Averill CA, Kalnins RM, Mitchell LA, Fabinyi GC, Jackson GD and Berkovic SF: Long-term seizure outcome and risk factors for recurrence after extratemporal epilepsy surgery. Epilepsia 53: 970-978, 2012.

43. Noachtar S, Bilgin O, Rémi J, Chang N, Midi I, Vollmar C and Feddersen B: Interictal regional polyspikes in noninvasive EEG suggest cortical dysplasia as etiology of focal epilepsies. Epilepsia 49: 1011-1017, 2008.
44. Kuruvilla A and Flink R: Intraoperative electrocorticography in epilepsy surgery: Useful or not? Seizure 12: 577-584, 2003.

45. Elsharkawy AE, Pannek H, Schulz R, Hoppe M, Pahs G, Gyimesi C, Nayel M, Issa A and Ebner A: Outcome of extratemporal epilepsy surgery experience of a single center. Neurosurgery 63: 516-525; discussion 525-526, 2008.

46. Kim DW, Kim HK, Lee SK, Chu K and Chung CK: Extent of neocortical resection and surgical outcome of epilepsy: Intracranial EEG analysis. Epilepsia 51: 1010-1017, 2010.

47. Poochikian-Sarkissian S, Sidani S, Wennberg R and Devins GM: Seizure freedom reduces illness intrusiveness and improves quality of life in epilepsy. Can J Neurol Sci 35: 280-286, 2008.

48. Jissendi-Tchofo P, Pandit F, Vallée L, Vinchon M, Pruvo JP, Baleriaux D and Soto Ares G: Brain regional glucose uptake changes in isolated cerebellar cortical dysplasia: Qualitative assessment using coregistrated FDG-PET/MRI. Cerebellum 11: 280-288, 2012

49. Ashjazadeh N, Yadollahikhales G, Ayoobzadehshirazi A, Sadraii N and Hadi N: Comparison of the health-related quality of life between epileptic patients with partial and generalized seizure. Iran J Neurol 13: 94-100, 2014. 